Arq. Bras. Med. Vet. Zootec., v.67, n.2, p.474-482, 2015

\title{
Glicerina bruta associada à silagem de sorgo em dietas para cordeiros
}

\author{
[Crude gliceryn associated with sorghum silagein diets for lambs] \\ C.A.A. Oliveira Filho ${ }^{1}$, J.A.G. Azevêdo ${ }^{2}$, G.G.P. Carvalho ${ }^{3}$, C.F.P.G. Silva ${ }^{4}$, J.D. Santos ${ }^{2}$ \\ ${ }^{1}$ Universidade Estadual do Sudoeste da Bahia - Itapetinga, BA \\ ${ }^{2}$ Universidade Estadual de Santa Cruz (UESC) - Ilhéus, BA \\ ${ }^{3}$ Universidade Federal da Bahia - Salvador, BA \\ ${ }^{4}$ Instituto Federal de Educação, Ciência e Tecnologia Baiano - Santa Inês, BA
}

\section{RESUMO}

Objetivou-se avaliar os níveis de glicerina bruta (GB), 0, 20, 40, 60 e $80 \mathrm{~g} / \mathrm{kg}$ de matéria seca (MS) de silagem de sorgo sobre a cinética da fermentação, $0,30,60$ e 90g/kg de MS de silagem sobre a cinética de degradação da fibra em detergente neutro (FDN), e os níveis $0,17,33,55$ e $70 \mathrm{~g} / \mathrm{kg}$ de MS de silagem sobre o consumo e a digestibilidade da MS, os compostos nutricionais e o balanço de nitrogênio em cordeiros. O volume e a taxa de degradação dos carboidratos não fibrosos (Vcnf) e (Kdenf), respectivamente, apresentaram comportamento quadrático, sendo estimado Vcnf máximo $(126,62 \mathrm{~mL} / \mathrm{g} \mathrm{de}$ MS) e Kdcnf mínima $(0,0782 / \mathrm{h})$ para inclusão de $54,85 \mathrm{~g}$ e $58,75 \mathrm{~g} \mathrm{~GB} / \mathrm{kg}$ de MS, respectivamente. Já o tempo de colonização (L), apresentou um decréscimo linear de $0,013 \mathrm{~h}$ para cada $10 \mathrm{~g}$ de $\mathrm{GB} / \mathrm{kg}$ de MS. Houve redução linear de $0,996 \%$ na degradação para fração B. Não houve efeito significativo da inclusão de GB sobre o consumo e a digestibilidade da MS, os compostos nutricionais e o balanço de nitrogênio. A utilização de GB entre 50 e $70 \mathrm{~g} / \mathrm{kg}$ de MS em dietas à base de silagem de sorgo pode ser fonte alternativa de energia para cordeiros.

Palavras-chave: alimento alternativo, coproduto, glicerol, valor nutricional,volumoso

\begin{abstract}
This study aimed to assess the levels of crude glycerin (CG) 0, 20, 40, 60 and $80 \mathrm{~g} / \mathrm{kg}$ of dry matter (DM) of sorghum silage on fermentation kinetics, $0,30,60$ and $90 \mathrm{~g} / \mathrm{kg}$ DM silage on the kinetics of degradation of neutral detergent fiber (NDF), and levels $0,17,33,55$ and $70 \mathrm{~g} / \mathrm{kg}$ DM silage on intake and digestibility of DM and nutritional compounds and nitrogen balance in lambs. The volume and rate of digestion of non-fiber carbohydrates (Vcnf) and (Kdcnf), respectively, showed a quadratic Vcnf being estimated maximum $(126.62 \mathrm{~mL} / \mathrm{g} \mathrm{DM})$ and Kdcnf minimum $(0.0782 / \mathrm{h})$ for inclusion of $54.85 \mathrm{CG}$ $58.75 \mathrm{~g} / \mathrm{kg} D \mathrm{DM}$, respectively. Since the time of colonization $(L)$, they presented a linear decrease of $0.013 \mathrm{~h}$

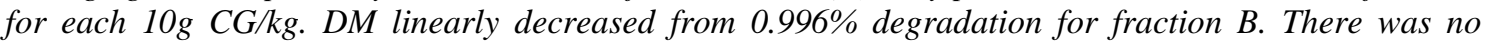
significant effect of the inclusion of $C G$ on the intake and dry matter digestibility and nutritional compounds and nitrogen balance. The use of $50 C G$ to $70 \mathrm{~g} / \mathrm{kg}$ DM in diets based on sorghum silage can be an alternative energy source for sheep.
\end{abstract}

Keywords: alternative feed, coproduct, glycerol, nutritional value, roughage

\section{INTRODUÇÃO}

A adoção de práticas de manejo que possibilitem reduções nos custos de produção, como o uso de alimentos energéticos alternativos que apresentem valor nutritivo adequado,

Recebido em 7 de fevereiro de 2014

Aceito em 9 de fevereiro de 2015

E-mail: carlos.uesb@hotmail.com disponibilidade e custo inferior aos alimentos convencionais como o milho, cujo preço oscila em função do mercado internacional, pode ser uma importante ferramenta para sustentabilidade econômica na produção de ruminantes.

Entre os coprodutos gerados pela cadeia produtiva do biodiesel com potencial de uso na 
alimentação de ruminantes, destaca-se a glicerina bruta (GB). A viabilidade do uso da GB como fonte de energia para ruminantes, além de gerar mais opções aos produtores, viabiliza um destino ecologicamente correto no aproveitamento desse coproduto.

No entanto, para que a utilização da GB se torne realidade, são necessárias pesquisas que resultem em respostas concernentes aos seus efeitos sobre a fermentação e a digestão dos alimentos no rúmen, à obtenção dos níveis ótimos de inclusão na dieta, ao desempenho e aos impactos na saúde dos animais alimentados com ela.

A simulação do ambiente ruminal e da digestão microbiana por meio da técnica semiautomática de produção de gases in vitro permite obter informações sobre a cinética de fermentação ruminal e conhecimento das taxas de degradação das frações de carboidratos fibrosos e não fibrosos, sendo essenciais para formulação de dietas que visam à máxima eficiência entre a utilização de fontes energéticas e proteicas, o que reflete diretamente no desempenho dos animais. A técnica ainda prevê informações sobre a extensão da digestão dos alimentos, às quais estão relacionadas diretamente ao potencial de aproveitamento dos compostos nutricionais pelos animais sob determinada condição (Maurício et al., 1999).

Diante do exposto, objetivou-se avaliar os efeitos de diferentes níveis de GB sobre a cinética de fermentação ruminal in vitro, a cinética de degradação in vitro da fibra em detergente neutro
(FDN), o consumo e a digestibilidade da matéria seca, os compostos nutricionais e o balanço de nitrogênio em cordeiros.

\section{MATERIAL E MÉTODOS}

O experimento foi conduzido no Laboratório de Pesquisa em Nutrição e Alimentação de Ruminantes (LaPNAR) do Departamento de Ciências Agrárias e Ambientais, na Universidade Estadual de Santa Cruz (UESC), Ilhéus-BA, durante os meses de março a junho de 2012.

Na produção da silagem de sorgo, foi utilizado o híbrido BRS 655 (Tab. 1). Para avaliação da cinética de fermentação, utilizou-se a técnica de produção dos gases in vitro de acordo com o descrito por Maurício et al. (1999). Em frascos de $50 \mathrm{~mL}$, foram adicionados $\mathrm{CO}_{2}, 0,3 \mathrm{~g}$ da amostra de silagem de sorgo e $28,125 \mathrm{~mL}$ de meio de cultura contendo minerais e tamponantes (Theodorou et al., 1994), associados com os níveis $0,20,40,60$ e $80 \mathrm{~g}$ de $\mathrm{GB} / \mathrm{kg}$ de $\mathrm{MS}$ da silagem de sorgo, com três réplicas para cada mistura.

As leituras de pressão dos gases produzidos durante as fermentações foram realizadas às zero, uma, duas, três, quatro, seis, oito, 10, 12, $14,16,18,20,24,28,36,48,60,72,84,96,108$, 120 e 144 horas após o início da incubação. As medidas de pressão obtidas foram ajustadas para o volume de gases conforme a equação estimada de Santos et al. (2010): V=0,04755+1,9754*p $+0,01407^{*} \mathrm{p}^{2}$, em que "V" é o volume dos gases $(\mathrm{mL})$ e "p" é a pressão dos gases dentro dos frascos de fermentação (psi).

Tabela 1. Composição química da silagem de sorgo e da glicerina bruta utilizadas no experimento

\begin{tabular}{|c|c|c|}
\hline Item & Silagem de sorgo & Glicerina bruta \\
\hline Matéria seca (g/kg) & 252,3 & 800,0 \\
\hline Matéria mineral (g/kg MS) & 51,2 & 35,0 \\
\hline Proteína bruta (g/kg MS) & 58,1 & - \\
\hline $\mathrm{FDNcp}^{1}(\mathrm{~g} / \mathrm{kg} \mathrm{MS})$ & 472,7 & - \\
\hline Lignina (g/kg MS) & 46,2 & - \\
\hline Extrato etéreo (g/kg MS) & 36,2 & 125,0 \\
\hline $\mathrm{CNF}^{2}(\mathrm{~g} / \mathrm{kg} \mathrm{MS})$ & 381,7 & - \\
\hline $\mathrm{MSi}^{3}(\mathrm{~g} / \mathrm{g})$ & 0,2 & - \\
\hline $\mathrm{FDNi}^{4}(\mathrm{~g} / \mathrm{g})$ & 0,2 & - \\
\hline $\operatorname{FDAi}^{5}(\mathrm{~g} / \mathrm{g})$ & 0,1 & - \\
\hline
\end{tabular}


A cinética da produção cumulativa dos gases foi avaliada empregando-se o modelo logístico bicompartimental para a estimativa dos parâmetros (Schofield et al., 1994): $\mathrm{V}(\mathrm{t})=(\mathrm{V} \operatorname{cnf} /(1+\exp (2-$ $\left.\left.4 * \mathrm{Kdcnf}^{*}(\mathrm{~T}-\mathrm{L})\right)\right)+\left(\mathrm{Vcf} /\left(1+\exp \left(2-4 * \mathrm{Kdcf}^{*}(\mathrm{~T}-\mathrm{L})\right)\right)\right.$, em que $\mathrm{V}(\mathrm{t})$ é o volume acumulado no tempo t; Vcnf, o volume de gás oriundo da fração de rápida digestão ou dos carboidratos não fibrosos (CNF); $\operatorname{Kdcnf}\left(\mathrm{h}^{-1}\right)$, a taxa de degradação da fração de rápida digestão ou dos $\mathrm{CNF}$; $\mathrm{L}$, a latência ou o tempo de colonização em horas; T, o tempo (h); Vcf, o volume dos gases da fração de lenta degradação (B2); Kdcf $\left(\mathrm{h}^{-1}\right)$, a taxa de degradação da fração B2. O volume máximo de produção dos gases (VT) foi obtido da seguinte forma: $V(t)=$ $\mathrm{V} c n f+\mathrm{Vcf}$.

Para avaliação da cinética de degradação da fibra em detergente neutro (FDN) (técnica gravimétrica), utilizou-se o mesmo modelo de incubação descrito para produção dos gases in vitro, adotando-se os tempos de retirada dos frascos zero, duas, quatro, oito, $12,24,48,60,72,96,120,144$ e 264 horas após incubação. Os tratamentos foram constituídos de 0,30, 60 e 90g de GB/kg de MS de silagem de sorgo.

Utilizou-se o modelo exponencial decrescente, corrigido para o período de latência descrito por Sampaio et al. (1995) para estimativa dos parâmetros: $\mathrm{Y}=\mathrm{A}-\mathrm{B} * \exp (-\mathrm{c} * \mathrm{t})$, em que: $\mathrm{Y}$ é o resíduo da FDN no tempo t; $\mathrm{A}$, a fração potencialmente degradável da FDN; $\mathrm{B}$, a fração insolúvel potencialmente degradável da FDN; exp, a base dos logaritmos neperianos; c, a taxa de degradação da fração B por unidade de tempo $\left(/ \mathrm{h}^{-1}\right)$; e t, o tempo de incubação. A fração indegradável (I) é igual a 100 - A.

No experimento in vivo, foram utilizados cinco cordeiros machos, sem raça definida, com peso médio inicial de $22,83 \pm 5,49 \mathrm{~kg}$, distribuídos casualmente em um delineamento em quadrado latino 5x5, sendo cinco níveis de GB e cinco períodos de coleta, de acordo com o modelo matemático: $\mathrm{Y}_{\mathrm{ij}(\mathrm{k})}=\mathrm{m}+\mathrm{P}_{\mathrm{i}}+\mathrm{A}_{\mathrm{j}}+\mathrm{t}_{\mathrm{k}}+\varepsilon_{\mathrm{ij}(\mathrm{k})}$, em que Yij = variável resposta; $\mathrm{m}=$ média geral; $\mathrm{P}_{\mathrm{i}}=$ efeito do período $\mathrm{i} ; \mathrm{A}_{\mathrm{j}}=$ efeito da animal $\mathrm{j} ; \mathrm{t}_{\mathrm{k}}=$ efeito do tratamento $\mathrm{k}$; e $\varepsilon_{\mathrm{ij}(\mathrm{k})}=$ erro experimental.

A utilização dos animais no experimento foi autorizada pela Comissão de Ética no Uso de Animais da Universidade Estadual de Santa Cruz, sob os protocolos 033, 034 e 035/2010. A cada animal ou unidade experimental destinou-se, casualmente, um dos cinco tratamentos, os quais foram constituídos por substituição de $0,20,40,60$ e $80 \mathrm{~g} / \mathrm{kg}$ da matéria natural da silagem de sorgo, representando, respectivamente, $0,17,35,53$ e $70 \mathrm{~g}$ de GB/kg de MS da silagem de sorgo (Tab. 2).

Tabela 2. Proporção de ingredientes e composição química das dietas em função dos níveis de glicerina bruta

\begin{tabular}{|c|c|c|c|c|c|}
\hline \multirow{2}{*}{ Composição } & \multicolumn{5}{|c|}{ Nível de glicerina bruta (g/kg MS) } \\
\hline & 0 & 17 & 35 & 53 & 70 \\
\hline \multicolumn{6}{|l|}{ Ingrediente (g/kg MS) } \\
\hline Silagem de sorgo & 978,6 & 961,3 & 944,0 & 926,3 & 908,9 \\
\hline Glicerina bruta & 0,0 & 17,4 & 35,0 & 52,8 & 70,4 \\
\hline Ureia & 12,8 & 12,5 & 12,3 & 12,1 & 11,9 \\
\hline Suplemento mineral $^{1}$ & 8,7 & 8,8 & 8,8 & 8,9 & 8,9 \\
\hline \multicolumn{6}{|l|}{ Composição química } \\
\hline Matéria seca $(\mathrm{g} / \mathrm{kg})$ & 268,2 & 277,6 & 287,1 & 296,8 & 306,3 \\
\hline Matéria orgânica (g/kg MS) & 928,5 & 912,1 & 895,6 & 878,9 & 862,4 \\
\hline Proteína bruta (g/kg MS) & 92,6 & 90,9 & 89,3 & 87,7 & 86,0 \\
\hline $\mathrm{FDNcp}^{2}(\mathrm{~g} / \mathrm{kg} \mathrm{MS})$ & 462,7 & 454,5 & 446,2 & 437,9 & 429,6 \\
\hline Carboidratos não fibrosos (g/kg MS) & 337,7 & 329,7 & 321,5 & 313,1 & 305 \\
\hline Extrato etéreo (g/kg MS) & 35,5 & 37,0 & 38,6 & 40,2 & 41,8 \\
\hline Fibra detergente ácido (g/kg MS) & 309,2 & 303,7 & 298,2 & 292,7 & 287,2 \\
\hline $\mathrm{PIDN}^{3}(\mathrm{~g} / \mathrm{kg} \mathrm{MS})$ & 12,7 & 12,5 & 12,2 & 12,0 & 11,8 \\
\hline $\mathrm{PIDA}^{4}(\mathrm{~g} / \mathrm{kg} \mathrm{MS})$ & 9,2 & 9,1 & 8,9 & 8,7 & 8,6 \\
\hline Lignina (g/kg MS) & 45,2 & 44,4 & 43,6 & 42,8 & 42,0 \\
\hline
\end{tabular}

(1) Composição (kg produto): Ca, 130-150g; P, 65g; Na, 130g; Mg, 10g; S, 12g; F, 650mg; Mn, 3000mg; Zn, 5000mg; Co, 80mg; Fe, 1000mg; Se, 10mg; I, 60mg. ${ }^{(2)} \mathrm{FDNcp}=$ fibra em detergente neutro corrigida para cinzas e proteínas. ${ }^{(3)} \mathrm{PIDN}=$ proteina insolúvel em detergente neutro; ${ }^{(4)} \mathrm{PIDA}=$ proteína insolúvel em detergente ácido. 
A duração total do experimento foi de 105 dias. Cada período experimental foi composto por 21 dias, sendo 16 dias de adaptação e cinco dias de coleta total. A coleta total de fezes foi realizada do $17^{\circ}$ ao $21^{\circ}$ dia, utilizando-se bolsas coletoras de couro sintético. As amostras de urina foram obtidas de todos os cordeiros por meio de coleta total a cada 24 horas em recipientes (baldes) no piso, contendo $100 \mathrm{~mL}$ de solução de ácido sulfúrico a $20 \% \mathrm{v} / \mathrm{v}$, durante os cinco dias de coletas do experimento.

As amostras das silagens fornecidas, das fezes e das sobras dos animais foram submetidas às análises de matéria seca (MS), proteína bruta $(\mathrm{PB})$, extrato etéreo (EE) e matéria mineral (MM) de acordo com a AOAC (Association..., 1990). Para análise de FDN, adotou-se a marcha analítica proposta por Nocek (1988); Mertens (1992); Licitra et al. (1996); Casali et al. (2009). O conteúdo de lignina foi obtido conforme Van Soest e Robertson (1985). O conteúdo de carboidratos não fibrosos (CNF), expresso em \% na MS, foi calculado segundo Hall (2000), como: $100-(\% \mathrm{FDN}+\% \mathrm{~PB}+\% \mathrm{EE}+\% \mathrm{MM})$

Para os cálculos de digestibilidade, adotou-se a fórmula: DAN g/g $=[(\mathrm{NCON}-\mathrm{NEXC}) / \mathrm{NCON}]$ $\mathrm{x} 100$, em que: $\mathrm{DAN}=$ coeficiente de digestibilidade aparente do nutriente; NCON = quantidade do nutriente consumido, em gramas; e NEXC = quantidade do nutriente excretado em gramas. O consumo de MS e compostos nutricionais foi obtido pela diferença entre o alimento ofertado e a sobra no final do período de 24 horas. $\mathrm{O}$ balanço dos compostos nitrogenados foi calculado pela diferença entre o nitrogênio ingerido (NI) e o nitrogênio excretado (urina e fezes).
As análises estatísticas para avaliação da cinética da fermentação e degradação in vitro foram conduzidas conforme um delineamento inteiramente ao acaso, composto de três repetições. Já em relação ao ensaio in vivo, as análises estatísticas foram conduzidas segundo um delineamento quadrado latino, composto de cinco repetições.

Para as variáveis cujo teste $\mathrm{F}$ foi significativo, a interpretação estatística dos efeitos dos níveis de substituição da GB foi feita por meio de análises de regressão. Todos os procedimentos estatísticos foram realizados com auxílio do programa estatístico SAS, atendendo todas as pressuposições relacionadas aos erros.

\section{RESULTADOS E DISCUSSÃO}

O volume e a taxa de degradação dos carboidratos não fibrosos (Vcnf e Kdcnf, respectivamente) bem como o tempo de latência (L) foram afetados significativamente $(\mathrm{P}<0,05)$ pela inclusão dos níveis de GB na cinética de fermentação ruminal in vitro (Tab. 3).

Verificou-se efeito linear decrescente $(\mathrm{P}<0,05)$ sobre o L com a inclusão de GB, observando-se um decréscimo de $0,013 \mathrm{~h}$ no $\mathrm{L}$ para cada 10 gramas de GB incluída na MS da silagem de sorgo. A inclusão dos níveis de GB favoreceu a colonização microbiana reduzindo o L (Tab. 3). Esses efeitos podem ser atribuídos ao aumento da disponibilidade de energia para o crescimento microbiano, uma vez que a GB apresenta, em média, 3,2Mcal de EM/kg de MS (Donkin, 2008).

Tabela 3. Estimativas dos parâmetros cinéticos de produção dos gases in vitro da silagem de sorgo com níveis de glicerina bruta, equações de regressão ajustadas e seus respectivos coeficientes de determinação

\begin{tabular}{|c|c|c|c|c|c|c|c|c|}
\hline \multirow{2}{*}{ Parâmetros } & \multicolumn{5}{|c|}{ Nível de glicerina bruta (g/kg MS) } & \multirow{2}{*}{$\begin{array}{l}\mathrm{EPM}_{ \pm}^{7} \\
\end{array}$} & \multirow{2}{*}{ Equação de regressão } & \multirow{2}{*}{$\mathrm{R}^{2} / \mathrm{r}^{2}$} \\
\hline & 0 & 20 & 40 & 60 & 80 & & & \\
\hline $\operatorname{Vcnf}^{1}\left(m L \cdot g^{-1}\right)$ & 97,98 & 108,82 & 132,64 & 121,05 & 121,58 & 3,80 & $\hat{\mathrm{Y}}=96,51+10,979 \mathrm{x}-1,0008 \mathrm{x}^{2}$ & 0,57 \\
\hline $\operatorname{Kdcnf}^{2}\left(h^{-1}\right)$ & 0,092 & 0,084 & 0,079 & 0,081 & 0,081 & 0,001 & $\hat{Y}=0,092-0,0047 x+0,0004 x^{2}$ & 0,67 \\
\hline $\mathrm{L}^{3}(\mathrm{~h})$ & 4,11 & 3,47 & 4,20 & 3,57 & 3,07 & 0,12 & $\hat{\mathrm{Y}}=3,9985-0,013 \mathrm{x}$ & 0,49 \\
\hline $\operatorname{Vcf}^{4}\left(\mathrm{~mL} \cdot \mathrm{g}^{-1}\right)$ & 134,57 & 125,86 & 126,63 & 119,48 & 123,49 & 2,24 & $\hat{\mathrm{Y}}=126,00$ & - \\
\hline $\operatorname{Kdcf}^{5}\left(h^{-1}\right)$ & 0,016 & 0,015 & 0,015 & 0,014 & 0,015 & 0,00 & $\hat{\mathrm{Y}}=0,015$ & - \\
\hline $\mathrm{VT}^{6}\left(\mathrm{~mL} \cdot \mathrm{g}^{-1} \mathrm{MS}\right)$ & 232,55 & 234,69 & 259,27 & 240,54 & 245,06 & 3,40 & $\hat{Y}=242,42$ & - \\
\hline
\end{tabular}

${ }^{(1)} \mathrm{Vcnf}=$ volume máximo de produção dos gases da fração dos $\mathrm{CNF} ;{ }^{(2)} \mathrm{Kdcnf}=$ taxa de digestão para a fração dos $\mathrm{CNF} ;{ }^{(3)} \mathrm{L}=$ tempo de colonização; ${ }^{(4)} \mathrm{Vcf}=$ volume máximo da produção de gases da fração dos $\mathrm{CF} ;{ }^{(5)} \mathrm{Kdcf}=$ taxa de digestão para a fração dos $\mathrm{CF} ;{ }^{(6)} \mathrm{VT}=$ volume máximo de produção de gases dos carboidratos totais; ${ }^{(7)} \mathrm{EPM}=$ erropadrão da média;* $\alpha=0.05$ de probabilidade para erro tipo $\mathrm{I}$. 
A observação de efeito significativo $(\mathrm{P}<0,05)$ para o L indica que a inclusão de GB em até $80 \mathrm{~g} / \mathrm{kg}$ MS não dificultou o acesso às células microbianas e, provavelmente, não alterou o sítio de ação das celulases dos microrganismos, assim como a afinidade pelo substrato. Os resultados obtidos na incubação utilizando a silagem de sorgo como substrato divergem dos relatados na literatura, em que a inclusão de glicerol aumentou o L nas incubações in vitro, tanto para forragens quanto para grãos (Pereira et al., 2008; Lee et al., 2011).

A diferença nos resultados obtidos em comparação à literatura pode estar relacionada ao período de adaptação dos microrganismos à GB e às características físicas, químicas e nutricionais dela, as quais variam de acordo com a fonte (origem vegetal ou animal) e o tipo de catálise empregada para sua obtenção, que reflete no grau de pureza, concentração de glicerol, ácidos graxos residuais não convertidos em biodiesel, minerais, álcool e água (Krehbiel, 2008). Neste trabalho, a GB utilizada foi oriunda de resíduo de óleo de cozinha, a sua pureza não foi avaliada e o fluido ruminal utilizado nas incubações foi obtido de dois bovinos não adaptados à GB.

Em relação ao volume dos gases produzidos e à taxa de digestão dos carboidratos não fibrosos (Vcnf e Kdcnf, respectivamente), a análise estatística revelou comportamento quadrático, sendo observado volume máximo produzido $(\mathrm{Vcnf}=126,62 \mathrm{~mL} / \mathrm{g}$ MS $)$ e taxa mínima (Kdenf $\left.=0,0782 . \mathrm{h}^{-1}\right)$ estimada para a inclusão de 54,85 e 58,75g de GB/kg de MS, respectivamente (Tab. $3)$.

Estes resultados indicam que a inclusão de aproximadamente 50,0g de $\mathrm{GB} / \mathrm{kg}$ de $\mathrm{MS}$ da silagem de sorgo pode ser benéfica aos microrganismos ruminais fermentadores de carboidratos não fibrosos, embora haja redução na velocidade da fermentação. O mecanismo de ação da GB (glicerol) sobre os microrganismos ruminais ainda é desconhecido, porém acreditase que torne o substrato menos acessível às células microbianas (Roger et al., 1992), o que pode explicar a menor taxa de fermentação encontrada.

De forma contrária, não houve efeito significativo $(\mathrm{P}>0,05)$ dos níveis de GB sobre a taxa de digestão (Kdcf) e o volume de produção dos gases a partir da fração fibrosa (Vcf) (Tab. 3 ), sendo observados valores médios de $\mathrm{Kdenf}=$ 0,015. $\mathrm{h}^{-1}$ e $\mathrm{Vcf}=126 \mathrm{~mL} / \mathrm{g}$ MS, respectivamente. As diferenças observadas no comportamento dos volumes e das taxas das frações fibrosas e não fibrosas indicam que os microrganismos ruminais apresentam diferenças na habilidade em se adaptar à $\mathrm{GB}$, o que indica a necessidade de pesquisas para avaliar quais os tipos de microrganismos ruminais, principalmente bactérias, que apresentam maior sensibilidade a níveis mais elevados de GB.

Não houve efeito significativo $(\mathrm{P}>0,05)$ dos níveis de GB sobre o VT (mL.g- $\left.{ }^{1} \mathrm{MS}\right)$, sendo observado valor médio de $242,42 \mathrm{~mL} .{ }^{1}{ }^{1} \mathrm{MS}$, respectivamente.

Já em relação aos parâmetros ajustados da cinética de degradação da FDN in vitro da silagem de sorgo, houve efeito significativo $(\mathrm{P}<0,05)$ apenas para a fração insolúvel potencialmente degradável da FDN (B), com um decréscimo de $0,996 \%$ na degradação para cada $10 \mathrm{~g}$ de $\mathrm{GB} / \mathrm{kg}$ de MS (Tab. 4). Essa observação indica que os microrganismos responsáveis por atuar sobre a fração B apresentaram maior sensibilidade à inclusão dos níveis de GB (glicerol).

Não houve efeito significativo $(\mathrm{P}>0,05)$ dos níveis de $\mathrm{GB}$ sobre a fração potencialmente degradável da FDN (A), a taxa de degradação da FDN (C) e a fração indigestível da FDN (I) da silagem de sorgo, estimando-se valores médios $80,63 \% ; 0,0343 \mathrm{~h}^{-1}$ e $19,35 \%$, respectivamente (Tab. 4). Essas observações demonstraram que a inclusão de GB em até $90 \mathrm{~g} / \mathrm{kg}$ de MS de silagem de sorgo não interferiu na atividade dos microrganismos que atuam sobre a fração potencialmente degradável da FDN (A), a taxa de degradação da FDN (C) e a fração indigestível da FDN (I) da silagem de sorgo. 
Tabela 4. Parâmetros médios ajustados relativos à cinética de degradação in vitro da fibra em detergente neutro da silagem de sorgo com níveis de glicerina bruta, equações de regressão ajustadas e seus respectivos coeficientes de determinação

\begin{tabular}{|c|c|c|c|c|c|c|c|}
\hline \multirow{2}{*}{ Parâmetros } & \multicolumn{4}{|c|}{$\begin{array}{l}\text { Nível de glicerina bruta } \\
\mathrm{g} / \mathrm{kg} \mathrm{MS}\end{array}$} & \multirow{2}{*}{$\underset{ \pm}{\mathrm{EPM}^{5}}$} & \multirow{2}{*}{$\begin{array}{l}\text { Equação de } \\
\text { regressão }\end{array}$} & \multirow[t]{2}{*}{$\mathrm{R}^{2} / \mathrm{r}^{2}$} \\
\hline & 0 & 30 & 60 & 90 & & & \\
\hline $\mathrm{A}^{1}(\%)$ & 80,17 & 80,56 & 81,29 & 80,57 & 0,21 & $y=80,64$ & - \\
\hline $\mathrm{B}^{2}(\%)$ & 33,78 & 30,87 & 27,54 & 24,93 & 1,28 & $y=33,7636-0,996 x$ & 0,96 \\
\hline $\mathrm{C}^{3}\left(/ \mathrm{h}^{-1}\right)$ & 0,02 & 0,03 & 0,02 & 0,02 & 0,001 & $\mathrm{y}=0,0343$ & - \\
\hline $\mathrm{I}^{4}(\%)$ & 19,83 & 19,44 & 18,71 & 19,43 & 0,21 & $y=19,35$ & - \\
\hline
\end{tabular}

${ }^{(1)} \mathrm{A}$ : degradação potencial da fração $\mathrm{FDN} ;{ }^{\left({ }^{2}\right)} \mathrm{B}$ : fração insolúvel potencialmente degradável; ${ }^{\left({ }^{3}\right)} \mathrm{C}$ : taxa de degradação da fração B por unidade de tempo; ${ }^{(4)} \mathrm{I}$ : fração indegradável da FDN; ${ }^{(5)} \mathrm{EPM}$ : erro-padrão médio; ${ }^{*} \alpha=0,05$ de probabilidade para o erro tipo I.

Em relação ao ensaio in vivo, a inclusão de GB em até $70 \mathrm{~g} / \mathrm{kg}$ MS na silagem de sorgo não afetou $(\mathrm{P}>0,05)$ o consumo de MS e de compostos nutricionais em cordeiros (Tab. 5). Os valores médios observados para o consumo de MS, MO FDNcp, PB, CNF, EE e NDT foram, respectivamente, 632,$3 ; 563,7 ; 276,8 ; 56,9$; 233,7; 24,80 e 383,7g/dia (Tab. 5).

Tabela 5. Consumos de fatores nutricionais nutrientes digestíveis totais (NDT), em cordeiros alimentados à base de silagem de sorgo e glicerina bruta

\begin{tabular}{|c|c|c|c|c|c|c|c|}
\hline \multirow{2}{*}{ Item } & \multicolumn{5}{|c|}{ Nível de glicerina bruta (g/kg MS) } & \multirow{2}{*}{$\begin{array}{c}\mathrm{EPM}^{4} \\
\pm\end{array}$} & \multirow{2}{*}{$\begin{array}{l}\text { Equação de } \\
\text { regressão }\end{array}$} \\
\hline & 0 & 17 & 33 & 55 & 70 & & \\
\hline $\begin{array}{l}\text { g/dia } \\
\text { Matéria seca }\end{array}$ & 683,56 & 623,26 & 638,96 & 588,58 & 627,02 & 39,5 & $\hat{\mathrm{Y}}=632,3$ \\
\hline Matéria orgânica & 634,80 & 567,66 & 570,40 & 513,74 & 532,12 & 36,7 & $\hat{\mathrm{Y}}=563,7$ \\
\hline Proteína bruta & 63,74 & 56,60 & 58,16 & 52,30 & 54,00 & 3,8 & $\hat{\mathrm{Y}}=56,9$ \\
\hline FDNcp $^{1}$ & 312,12 & 277,58 & 279,56 & 250,78 & 264,14 & 16,1 & $\hat{\mathrm{Y}}=276,8$ \\
\hline $\mathrm{CNF}^{2}$ & 262,00 & 235,60 & 235,10 & 213,90 & 222,00 & 16,9 & $\hat{Y}=233,7$ \\
\hline Extrato etéreo & 24,28 & 24,30 & 26,20 & 24,56 & 24,86 & 2,1 & $\hat{\mathrm{Y}}=24,80$ \\
\hline $\mathrm{NDT}^{3}$ & 434,90 & 381,00 & 392,50 & 347,80 & 362,20 & 33,5 & $\hat{\mathrm{Y}}=383,7$ \\
\hline Matéria seca $\mathrm{g} / \mathrm{kg} \mathrm{PC}$ & 28,55 & 26,52 & 26,44 & 26,43 & 26,62 & 1,3 & $\hat{\mathrm{Y}}=26,9$ \\
\hline Matéria seca $\mathrm{g} / \mathrm{kg}^{0,75}$ & 63,04 & 58,22 & 58,39 & 57,28 & 58,46 & 3,0 & $\hat{\mathrm{Y}}=59,1$ \\
\hline $\mathrm{FDNcp}^{1} \mathrm{~g} / \mathrm{kg} \mathrm{PC^{5 }}$ & 13,05 & 11,85 & 11,61 & 11,26 & 11,23 & 0,6 & $\hat{\mathrm{Y}}=11,8$ \\
\hline FDNcp $^{1} \mathrm{~g} / \mathrm{kg}^{0,75}$ & 28,79 & 25,99 & 25,62 & 24,41 & 24,66 & 1,2 & $\hat{\mathrm{Y}}=25,9$ \\
\hline
\end{tabular}

${ }^{(1)}$ FDNcp: fibra em detergente neutro corrigida para cinzas e proteínas; ${ }^{(2)} \mathrm{CNF}$ : carboidratos não fibrosos; ${ }^{(3)} \mathrm{NDT}$ : nutrientes digestíveis totais. ${ }^{(4)} \mathrm{EMP}$ : erro-padrão da média; ${ }^{(5)} \mathrm{PC}$ : peso corporal; ${ }^{*} \alpha=0,05$ de probabilidade para erro tipo I.

O efeito não significativo $(\mathrm{P}>0,05)$ dos níveis de GB sobre o consumo de MS e a proporção semelhante dos compostos nutricionais nas dietas (Tab. 2) podem explicar o efeito não significativo $(\mathrm{P}>0,05)$ dos níveis de GB sobre o consumo de MO, FDNcp, PB, CNF, EE e NDT. Desta forma, os resultados observados estão mais associados com as características nutricionais da silagem de sorgo.

Machado et al. (2011) avaliaram o consumo e a digestibilidade aparente de três híbridos, BRS 610, BR 700 e BRS 655, colhidos em três estádios de maturação dos grãos, leitoso, pastoso e farináceo, e observaram valores médios para o consumo de MS entre 45,90 e 51,20g/kg,75, para híbrido BRS 655, o mesmo utilizado neste trabalho. O valor médio obtido para o consumo de MS, $59,07 \mathrm{~g} / \mathrm{kg}^{0,75}$, foi superior ao observado por Machado et al. (2011). Tal fato pode ser explicado pela diferença na composição química das silagens. Neste trabalho, a silagem de sorgo utilizada apresentou valores médios de 446,18 e 43,6g/kg MS para FDNcp e lignina, respectivamente, inferiores aos observados por 
Machado et al. (2011) (629,5 e 58,5g/kg MS, respectivamente).

A inclusão de GB em até $70 \mathrm{~g} / \mathrm{kg}$ de $\mathrm{MS}$ na silagem de sorgo não afetou a proporção de MS e compostos nutricionais absorvidos pelo organismo dos animais, o que pode ser confirmado pelo efeito não significativo $(\mathrm{P}>0,05)$ dos níveis de GB sobre os coeficientes de digestibilidade aparente da MS, MO, FDNcp, CNF, PB e EE (Tab. 6). Os valores médios observados para os coeficientes de digestibilidade aparente da MS, MO, FDNcp, $\mathrm{PB}, \mathrm{CNF}$ e EE foram, respectivamente, 0,59; 0,$57 ; 0,42 ; 0,56 ; 0,85$ e $0,78 \mathrm{~g} / \mathrm{g}$ (Tab. 6).

Tabela 6. Coeficientes de digestibilidade aparente de fatores nutricionais em cordeiros alimentados com silagem de sorgo associados com níveis de glicerina bruta

\begin{tabular}{|c|c|c|c|c|c|c|c|}
\hline \multirow{2}{*}{ Digestibilidade } & \multicolumn{5}{|c|}{ Nível de glicerina bruta (g/kg MS) } & \multirow{2}{*}{$\begin{array}{c}\mathrm{EPM}^{4} \\
\pm\end{array}$} & \multirow{2}{*}{ Equação de regressão } \\
\hline & 0 & 17 & 33 & 55 & 70 & & \\
\hline Matéria seca $(\mathrm{g} / \mathrm{g})$ & 0,59 & 0,57 & 0,60 & 0,60 & 0,61 & 0,01 & $\hat{\mathrm{Y}}=0,59$ \\
\hline Matéria orgânica (g/g) & 0,59 & 0,56 & 0,58 & 0,57 & 0,58 & 0,01 & $\hat{\mathrm{Y}}=0,57$ \\
\hline $\operatorname{FDNcp}^{1}(\mathrm{~g} / \mathrm{g})$ & 0,44 & 0,43 & 0,42 & 0,43 & 0,39 & 0,02 & $\hat{\mathrm{Y}}=0,42$ \\
\hline $\mathrm{CNF}^{2}(\mathrm{~g} / \mathrm{g})$ & 0,84 & 0,85 & 0,88 & 0,87 & 0,83 & 0,01 & $\hat{\mathrm{Y}}=0,85$ \\
\hline Proteína bruta (g/g) & 0,61 & 0,50 & 0,54 & 0,52 & 0,60 & 0,02 & $\hat{\mathrm{Y}}=0,56$ \\
\hline Extrato etéreo $(\mathrm{g} / \mathrm{g})$ & 0,80 & 0,79 & 0,76 & 0,78 & 0,79 & 0,02 & $\hat{\mathrm{Y}}=0,78$ \\
\hline $\mathrm{NDT}^{3}(\mathrm{~g} / \mathrm{g})$ & 0,66 & 0,64 & 0,63 & 0,62 & 0,58 & 0,01 & $\hat{\mathrm{Y}}=0,63$ \\
\hline
\end{tabular}

${ }^{(1)}$ FDNcp: fibra em detergente neutro corrigida para cinzas e proteína; ${ }^{(2)} \mathrm{CNF}$ : carboidratos não fibrosos; ${ }^{(3)} \mathrm{NDT}$ : nutrientes digestíveis totais; ${ }^{(4)} \mathrm{EPM}$ : erro-padrão da média; $* \alpha=0,05$ de probabilidade para erro tipo I.

Os valores médios observados para os coeficientes de digestibilidade aparente da MS e da $\mathrm{MO}$, respectivamente 0,59 e $0,57 \mathrm{~g} / \mathrm{g}$, estão dentro da faixa obtida por Souza et al. (2003), cujos valores variaram entre 0,50 e $0,64 \mathrm{~g} / \mathrm{g}$ para a digestibilidade da MS e entre 0,53 e $0,65 \mathrm{~g} / \mathrm{g}$ para a digestibilidade da $\mathrm{MO}$, avaliando-se o valor nutritivo de silagens de diferentes híbridos de sorgo de porte médio e alto.

Os valores médios observados para os coeficientes de digestibilidade aparente da FDNcp e MS (0,42 e 0,59g/g, respectivamente) apresentaram-se semelhantes aos observados por Machado et al. (2011) para o híbrido BRS 655, nos estádios pastoso e farináceo (entre 0,43 e $0,39 \mathrm{~g} / \mathrm{g}$ e 0,52 e $0,49 \mathrm{~g} / \mathrm{g}$ ) para os coeficientes de digestibilidade aparente da FDNcp e MS, respectivamente. $\mathrm{O}$ estádio de maturação na colheita, a composição química das silagens, as condições climáticas, a relação colmo:panícula das plantas e as variações entre os animais experimentais, entre outros fatores, podem explicar as diferenças entre os resultados.

Não houve efeito significativo $(\mathrm{P}>0,05)$ dos níveis de GB sobre o $\mathrm{N}$ (g/animal/dia) consumido, fecal, urinário e retido (Tab. 7). A observação de efeito não significativo $(\mathrm{P}>0,05)$ dos níveis $0,17,33,55$ e $70 \mathrm{~g} / \mathrm{kg}$ de $\mathrm{MS}$ de silagem de sorgo sobre a ingestão de $\mathrm{N}$ $(9,11 \mathrm{~g} / \mathrm{dia})$, a excreção fecal $(3,96 \mathrm{~g} / \mathrm{dia})$ e a urinária $(2,14)$, resultando em balanço positivo de $\mathrm{N}$ (3,01g/dia) (Tab. 7), leva a inferir que houve equilíbrio e sincronia de degradação das fontes proteicas e energéticas das dietas uma vez que a relação proteína:energia não afetou a retenção de nitrogênio.

Tabela 7. Valores médios diários do balanço de nitrogênio em cordeiros alimentados com silagem de sorgo associado com níveis de glicerina bruta

\begin{tabular}{|c|c|c|c|c|c|c|c|}
\hline \multirow{2}{*}{ Nitrogênio } & \multicolumn{5}{|c|}{ Nível de glicerina bruta (g/kg MS) } & \multirow{2}{*}{$\begin{array}{c}\text { EPM }^{1} \\
\pm\end{array}$} & \multirow{2}{*}{$\begin{array}{l}\text { Equação de } \\
\text { regressão }\end{array}$} \\
\hline & 0 & 17 & 33 & 55 & 70 & & \\
\hline Consumido (g/dia) & 10,20 & 9,05 & 9,31 & 8,36 & 8,64 & 0,62 & $\hat{\mathrm{Y}}=9,11$ \\
\hline Fecal (g/dia) & 3,76 & 4,31 & 4,17 & 3,96 & 3,60 & 0,26 & $\hat{\mathrm{Y}}=3,96$ \\
\hline Urinário (g/dia) & 2,54 & 2,20 & 1,97 & 2,12 & 1,87 & 0,16 & $\hat{Y}=2,14$ \\
\hline Retido (g/dia) & 3,90 & 2,54 & 3,17 & 2,28 & 3,17 & 0,44 & $\hat{\mathrm{Y}}=3,01$ \\
\hline
\end{tabular}

${ }^{(1)}$ EPM: erro-padrão da média; ${ }^{*} \alpha=0,05$ de probabilidade para o erro tipo I. 
O aumento de glicerol com a inclusão dos níveis de GB, provavelmente, não afetou o crescimento das bactérias e, consequentemente, a retenção de $\mathrm{N}$, visto que, com a elevação da quantidade de GB, não foram observadas diferenças na retenção de $\mathrm{N}(\mathrm{P}>0,05)$. A observação de efeito não significativo $(\mathrm{P}>0,05)$ dos níveis de GB sobre o consumo de MS (Tab. 5) e a semelhança na concentração de PB entre as dietas (Tab. 2) podem explicar o efeito não significativo $(\mathrm{P}>0,05)$ no balanço de $\mathrm{N}$.

Em relação à excreção de $\mathrm{N}$, o $\mathrm{N}$ perdido via urina $(2,14 \mathrm{~g} / \mathrm{dia})$ não superou a excreção de $\mathrm{N}$ fecal $(3,96 \mathrm{~g} / \mathrm{dia})$. Dois fatores podem ter influenciado esse resultado. O primeiro refere-se ao fato de que a fonte de nitrogênio predominante nas dietas à base de sorgo foi a proteína verdadeira com menor contribuição de ureia (Tab. 1), o que minimizou as perdas de amônia no rúmen. O segundo fator seria a presença de tanino no cultivar utilizado.

\section{CONCLUSÕES}

A utilização de glicerina bruta entre 50 e $70 \mathrm{~g} / \mathrm{kg}$ de MS em dietas à base de silagem de sorgo pode ser fonte alternativa de energia para cordeiros, já que nessas proporções resulta em maior produção dos gases oriunda da fração dos carboidratos não fibrosos, além de não afetar o consumo e a digestibilidade dos nutrientes bem como o balanço de compostos nitrogenados.

\section{AGRADECIMENTOS}

Os autores agradecem ao CNPq (MCT/CNPq processo $\mathrm{n}^{\circ}$. 475129/2010-5), à Universidade Estadual de Santa Cruz, à Fundação de Amparo à Pesquisa do Estado da Bahia e ao Banco do Nordeste do Brasil, pelo suporte financeiro.

\section{REFERÊNCIAS}

ASSOCIATION Official Analytical Chemists (AOAC). Official methods of analysis.16.ed. Washington, D.C.: AOAC, 1990. 1094p.

CASALI, A.O.; DETMANN, E.; VALADARES FILHO, S.C. et al. Estimação de teores de componentes fibrosos em alimentos para ruminantes em sacos de diferentes tecidos. Rev. Bras. Zootec., v.38, p.130-138, 2009.
DONKIN, S.S. Glicerol from biodiesel production: the new corn for dairy cattle. Rev. Bras. Zootec., v.37, p.280-286, 2008.

HALL, M.B. Calculation of non-structural carbohydrate content of feeds that contain nonprotein nitrogen.University of Florida. Bulletin 339, p.A-25. 2000.

KREHBIEL, C.R. Ruminal and physiological metabolsim of glycerin. J. Anim. Sci., v.2, p.392, 2008.

LEE, S.Y.; LEE, S.M.; CHO, Y.B. et al. Glycerol as a feed suplementation for ruminants: In vitro fermentation characteristic and methane production. Anim. Feed Sci. Technol., v.166, p.269-274, 2011.

LICITRA, G.; HERNANDEZ, T.M.; VAN SOEST, P.J. Standardization of procedures for nitrogen fractionation of ruminant feeds. Anim. Feed Sci. Technol., v.57, p.347-358, 1996.

MACHADO, F.S.; RODRÍGUEZ, N.M.; GONÇALVES, L.C. et al. Consumo e digestibilidade aparente de silagens de sorgo em diferentes estádios de maturação. Arq. Bras. Med. Vet. Zootec., v.63, p.1470-1478, 2011.

MAURICIO, R.M.; MOULD, F.L.; DHANOA, M.S. et al. A semi-automated in vitro gas production technique for ruminant feedstuff evaluation. Anim. Feed Sci. Technol., v.79, p.321-330, 1999.

MERTENS, D.R. Análise da fibra e sua utilização na avaliação de alimentos e formulação de rações. In: SIMPÓSIO INTERNACIONAL DE RUMINANTES, 1992, Lavras. Anais... Lavras: Sociedade Brasileira de Zootecnia, 1992. p.188-219. (Resumo)

NOCEK, J.E. In situ and other methods to estimate ruminal protein and energy digestibility. A review. J. Dairy Sci., v.71, p.2051-2069, 1988.

PEREIRA, L.G.R.; MAURÍCIO, R.M.; MENEZES, D.R. et al. Influência da glicerina bruta na cinética de fermentação ruminal in vitro. In: REUNIÃO ANUAL DA SOCIEDADE BRASILEIRA DE ZOOTECNIA, 45., 2008, Lavras. Anais... Lavras: [s.n.], 2008. (Resumo).

ROGER, V.; FONTY, G.; ANDRE, C.; GOUET, $\mathrm{P}$. Effects of glycerol on the growth, adhesion, and cellulolytic activity of rumen cellulolytic bacteria and anaerobic fungi. Cur. Microb., v.25, p.197-201, 1992. 
SANTOS, M.G.M.F.; AZEVÊDO, J.A.G.; PEREIRA, L.G.R. et al. Relação entre pressão e volume para implantação da técnica in vitro de produção de gases no trópico úmido. In: REUNIÃO ANUAL DA SOCIEDADE BRASILEIRA DE ZOOTECNIA, 47., Salvador. Anais... Salvador: [s.n.] 2010. (Resumo).

SAMPAIO, I.B.M.; PIKE, D.J.; OWEN, E. Optimal design for studying dry matter degradation in the rumen. Arq. Bras. Med. Vet. Zootec., v.47, p.373-383, 1995.

SCHOFIELD, P.; PITT, R.E.; PELL, A.N. Kinetcs of fiber digestion from in vitro gas production. J. Anim. Sci., v.72, p.2980-2991, 1994.
SOUZA, V.G.; PEREIRA, O.G.; DE MORAES, S.A. et al. Valor Nutritivo de Silagens de Sorgo. Rev. Bras. Zootec., v.32, p.753-759, 2003.

THEODOROU, M.K.; WILLIAMS, B.A.; DHANOA, M.S. et al. A simple gas production method using a pressure transducer to determine fermentation kinetics of ruminant feeds. Anim. Feed Sci. Technol., v.48, p.185-197, 1994.

VAN SOEST, P.J.; ROBERTSON, J.B. (Ed) Analysis of forages and fibrous foods. Ithaca: CORNELL UNIVERSITY, 1985. 202p. 